\title{
Dynamics of a Controlled Articulated $n$-trailer Wheeled Vehicle
}

\section{E. A. Mikishanina}

This article is devoted to the study of the dynamics of movement of an articulated $n$-trailer wheeled vehicle with a controlled leading car. Each link of the vehicle can rotate relative to its point of fixation. It is shown that, in the case of a controlled leading car, only nonholonomic constraint equations are sufficient to describe the dynamics of the system, which in turn form a closed system of differential equations. For a detailed analysis of the dynamics of the system, the cases of movement of a wheeled vehicle consisting of three symmetric links are considered, and the leading link (leading car) moves both uniformly along a circle and with a modulo variable velocity along a certain curved trajectory. The angular velocity remains constant in both cases. In the first case, the system is integrable and analytical solutions are obtained. In the second case, when the linear velocity is a periodic function, the solutions of the problem are also periodic. In numerical experiments with a large number of trailers, similar dynamics are observed.

Keywords: wheeled vehicle, nonholonomic constraint, mathematical model, dynamics of system

\section{Introduction}

Trends in modern industry are such that, in the production process, preference is increasingly given to robotic devices. In connection with the development of so-called "smart" production, there is a need for rational transportation of goods without human intervention. This problem is an integral part of modern production and is solved by the introduction of autonomous transport robots. They could automate the process of transporting goods and still make it safe even without human intervention.

Received December 08, 2020

Accepted March 16, 2021

Evgeniya A. Mikishanina

evaeva_84@mail.ru

Chuvash State University

Moskovskii prosp. 15, Cheboksary, 428015 Russia 
One of the first works on the dynamics of wheeled vehicles is that of Rocard [1]. Later, more extensive research in this area was carried out in the work of L. G. Lobas [2], who presented the results of research on the dynamical properties of mechanical rolling systems that model cars, motorcycles and intrafactory carts. However, the first works were characterized mainly by the complexity of the statements and calculations. Modern works on the dynamics of wheeled vehicles are initiated by the development of the robotic sphere $[3,4]$ and by the need to solve the problem of controlling $n$-trailer vehicles $[5,6]$.

The works [7-10] are devoted to the movement of wheeled vehicles on a plane. Wheeled vehicle designs can be very diverse. The issue of kinematics and dynamics of an articulated $n$-trailer vehicle is considered in the most meaningful way in [7]. In that paper, the authors provide a derivation of the equations of motion based on the method proposed in [11]. A vehicle with two wheel pairs is considered in [8] and a vehicle with two symmetric links is considered in [9]. A nonholonomic model of a snakeboard, consisting of three links and two wheel pairs that are attached to the platform, is presented in [10]. This model can be regarded as a generalization of the classical nonholonomic model of the Chaplygin sleigh [12]. A discussion of the kinematics of wheeled vehicles can also be found in [13-15].

In this paper, we consider another model of an articulated $n$-trailer wheeled vehicle consisting of an arbitrary number of links. The links hinged to each other contain one wheel pair and can rotate around their points of fixation with other parts, and the mass centers of the links coincide with the geometric centers of the links and the geometrical centers of the wheel pairs. This model is slightly different from the models that were considered in the above-mentioned works. However, a similar design is found in [16]. It is also assumed that the leading car moves in a prescribed manner, that is, the system is under control. The work on the dynamics of an uncontrollable wheeled vehicle [17] was published by the authors earlier. To describe the dynamics of a nonholonomic system, it is necessary to solve the equations of motion written in the Lagrange form with undetermined multipliers [18]. In the case of a controlled leading car, it is shown that only the use of nonholonomic constraint equations is sufficient to describe the dynamics of the entire vehicle.

This paper also considers special cases of movement of three-link and four-link wheeled vehicles with constant angular velocity. As shown by numerical experiments, with a larger number of trailers, similar dynamics is observed. Analytical solutions are obtained for the case of uniform movement of the leading car in a circle. Graphs of numerical solutions are plotted in the case of uneven movement of the leading car along a given curved path. Also, graphs of numerical solutions are presented.

\section{Mathematical model of a mobile wheeled vehicle}

Consider a system of $(n+1)$ articulated links (a leading car and trailers) moving on a plane. For convenience, we will call the leading link zero, the next link first, and so on. Each link contains one wheel pair. The links are pivotally attached to each other and can rotate around their attachment points. Assume that the centers of mass of each element of the structure are at their geometric centers and the geometric centers of the wheel pairs and lie on the same axis with the points of attachment of this element with neighboring elements. For convenience, we will consider the leading link to be zero, the next link will be considered the first, and so on.

The coordinates of the center of mass of each link are denoted by $\left(x_{i}, y_{i}\right)$. The coordinates of the points $O_{i}$ of the attachment of the $(i-1)$ th and the $i$ th element are denoted $\left(X_{i}, Y_{i}\right)$. 


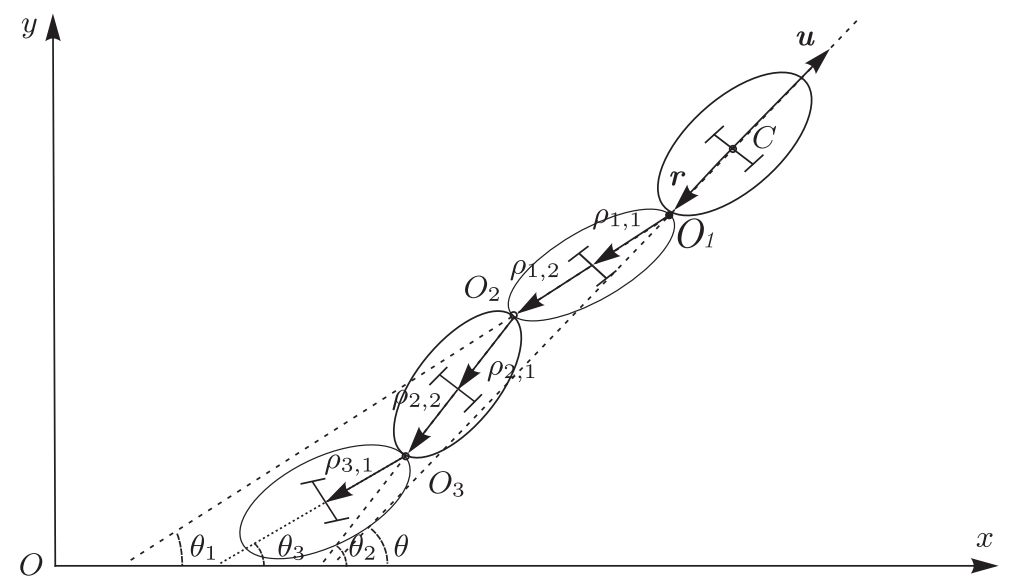

Fig. 1. Example of a four-link wheeled vehicle.

The vector connecting the center of mass of the leading element to the point $O_{1}$ is denoted by $\boldsymbol{r}$, the vector connecting the point $O_{i}$ to the center of mass of the $k$ th link is denoted by $\boldsymbol{\rho}_{i, 1}$ and the vector connecting the center of mass of the $i$ th element to the point of attachment of the elements $O_{i+1}$ is denoted by $\boldsymbol{\rho}_{i, 2}$. The identity $\boldsymbol{\rho}_{i}=\boldsymbol{\rho}_{i, 1}+\boldsymbol{\rho}_{i, 2}$ is also valid.

Let us define the lengths of the introduced vectors:

$$
|\boldsymbol{r}|=a,\left|\boldsymbol{\rho}_{i, 1}\right|=a_{i, 1},\left|\boldsymbol{\rho}_{i, 2}\right|=a_{i, 2},\left|\boldsymbol{\rho}_{i}\right|=a_{i} .
$$

Let us denote the angle between the axis of the leading link and axis $O x$ by $\theta$, which is the angle between the vector $-\boldsymbol{r}$ and the positive direction of the axis $O x$, the angle between the axis of the $i$ th link (vector $-\boldsymbol{\rho}_{i}$ ) and the positive direction of the axis $O x$ by $\theta_{i}$, and the angle between the axes of the $(i-1)$ th element and the $i$ th element (the angle of rotation from vector $-\boldsymbol{\rho}_{i-1}$ to vector $\left.\boldsymbol{\rho}_{i}\right)$ by $\alpha_{i}$ and $\alpha_{i}, \theta_{i} \in[-\pi, \pi)$. Then the angular velocity of the zero (leading) link is $\dot{\theta}=\omega$. For the other links the following applies:

$$
\theta_{i}=\theta+\sum_{k=1}^{i} \alpha_{k}, \quad \dot{\theta}_{i}=\omega_{i}
$$

Then the coordinates of the centers of mass $\left(x_{i}, y_{i}\right)$ of each element can be calculated using the formula

$$
\begin{aligned}
& x_{i}=x_{0}-a \cos \theta-\sum_{k=1}^{i-1} a_{k} \cos \theta_{k}-a_{i, 1} \cos \theta_{i}, \\
& y_{i}=y_{0}-a \sin \theta-\sum_{k=1}^{i-1} a_{k} \sin \theta_{k}-a_{i, 1} \sin \theta_{i},
\end{aligned}
$$

where $\left(x_{0}, y_{0}\right)$ are the coordinates of the center of mass of the leading car. We assume that $\sum_{k=1}^{0} f_{k}=0$.

The kinetic energy of the leading car has the form

$$
T_{0}=\frac{1}{2}\left(I_{0} \omega^{2}+m_{0}\left(\dot{x}_{0}^{2}+\dot{y}_{0}^{2}\right)\right),
$$


where $I_{0}$ is the moment of inertia of the first link relative to the center of mass and $m_{0}$ is the hollow mass of the first link. The kinetic energy of the $i$ th link has the form

$$
T_{i}=\frac{1}{2}\left(I_{i} \dot{\theta}_{i}^{2}+m_{i}\left(\dot{x}_{i}^{2}+\dot{y}_{i}^{2}\right)\right),
$$

where $I_{i}$ is the moment of inertia of the $i$ th link relative to the center of mass and $m_{i}$ is the hollow mass of the $i$ th link. The kinetic energy of the system will take the form

$$
T=\frac{1}{2}\left(I_{0} \omega^{2}+m_{0}\left(\dot{x}_{0}^{2}+\dot{y}_{0}^{2}\right)+\sum_{i=1}^{n} I_{i} \dot{\omega}_{i}^{2}+\sum_{i=1}^{n} m_{i}\left(\dot{x}_{i}^{2}+\dot{y}_{i}^{2}\right)\right) .
$$

Calculate the derivatives in equations (1.1)

$$
\begin{aligned}
& \dot{x}_{i}=\dot{x}_{0}+a \omega \sin \theta+\sum_{k=1}^{i-1} a_{k} \omega_{k} \sin \theta_{k}+a_{i, 1} \omega_{i} \sin \theta_{i}, \\
& \dot{y}_{i}=\dot{y}_{0}-a \omega \cos \theta-\sum_{k=1}^{i-1} a_{k} \omega_{k} \cos \theta_{k}-a_{i, 1} \omega_{i} \cos \theta_{i} .
\end{aligned}
$$

Let us introduce the following assumption for convenience: $m_{i}=m, i=\overline{0, n}$. Calculate the following sum:

$$
\begin{aligned}
\sum_{i=1}^{n}\left(\dot{x}_{i}^{2}+\dot{y}_{i}^{2}\right) & =n\left(\dot{x}_{0}^{2}+\dot{y}_{0}^{2}+a^{2} \omega^{2}\right)+2 a n \omega\left(\dot{x}_{0} \sin \theta-\dot{y}_{0} \cos \theta\right)+ \\
& +\sum_{k=1}^{n}(n-k) a_{k}^{2} \omega_{k}^{2}+\sum_{k=1}^{n} a_{k, 1}^{2} \omega_{k}^{2}+ \\
& +2 \sum_{k=1}^{n}(n-k) a_{k} \omega_{k}\left(\dot{x}_{0} \sin \theta_{k}-\dot{y}_{0} \cos \theta_{k}\right)+2 \sum_{k=1}^{n} a_{k, 1} \omega_{k}\left(\dot{x}_{0} \sin \theta_{k}-\dot{y}_{0} \cos \theta_{k}\right)+ \\
& +2 a \omega \sum_{k=1}^{n}(n-k) a_{k} \omega_{k} \cos \left(\theta_{k}-\theta\right)+2 a \omega \sum_{k=1}^{n} a_{k, 1} \omega_{k} \cos \left(\theta_{k}-\theta\right)+ \\
& +2 \sum_{k=2}^{n} \sum_{i=1}^{k-1} a_{i} a_{k, 1} \omega_{i} \omega_{k} \cos \left(\theta_{k}-\theta_{i}\right)+2 \sum_{k=2}^{n} \sum_{i=1}^{k-1}(n-k) a_{i} a_{k} \omega_{i} \omega_{k} \cos \left(\theta_{k}-\theta_{i}\right) .
\end{aligned}
$$

After the substitution $A_{k}=(n-k) a_{k}+a_{k, 1}, B_{k}=(n-k) a_{k}^{2}+a_{k, 1}^{2}$, the kinetic energy can be represented as

$$
\begin{aligned}
T & =\frac{1}{2}\left(\left(I_{0}+m n a^{2}\right) \omega^{2}+m(n+1)\left(v_{1}^{2}+v_{2}^{2}\right)+2 m n a \omega\left(v_{1} \sin \theta-v_{2} \cos \theta\right)+\right. \\
& \left.+\sum_{k=1}^{n}\left(I_{k}+m B_{k}\right) \omega_{k}^{2}\right)+m\left[\sum_{k=1}^{n} A_{k} \omega_{k}\left(v_{1} \sin \theta_{k}-v_{2} \cos \theta_{k}\right)+\right. \\
& \left.+a \omega \sum_{k=1}^{n} A_{k} \omega_{k} \cos \left(\theta_{k}-\theta\right)+\sum_{k=2}^{n} \sum_{i=1}^{k-1} A_{k} a_{i} \omega_{k} \omega_{i} \cos \left(\theta_{k}-\theta_{i}\right)\right]
\end{aligned}
$$

where $\dot{x}_{0}=v_{1}, \dot{y}_{0}=v_{2}$ are the components of the linear velocity. For further modeling, it is necessary to formulate equations of motion in Lagrangian form and equations with a nonholonomic constraint. 


\section{Equations of motion}

Let the leading car be under control, i.e., let the components of the velocity $v_{1}(t), v_{2}(t)$ be known. Then one can determine the value of $\theta$ from the equation

$$
v_{1} \sin \theta-v_{2} \cos \theta=0
$$

using the standard ATAN2 function

$$
\theta(t)=\operatorname{atan} 2\left(v_{2}(t), v_{1}(t)\right), \quad \theta \in[-\pi, \pi) .
$$

Then the expression for the angular velocity takes the form

$$
\omega(t)=\frac{\dot{v_{2}} v_{1}-\dot{v_{1}} v_{2}}{v_{1}^{2}+v_{2}^{2}}
$$

The equations of motion for a controlled $n$-trailer wheeled vehicle have the form

$$
\frac{d}{d t}\left(\frac{\partial T}{\partial \omega_{k}}\right)-\frac{\partial T}{\partial \theta_{k}}=\lambda_{1} \frac{\partial f_{1}}{\partial \omega_{k}}+\ldots+\lambda_{n} \frac{\partial f_{n}}{\partial \omega_{k}}, \quad k=\overline{1, n}
$$

where the equations

$$
f_{i}=\dot{x}_{i} \sin \theta_{i}-\dot{y}_{i} \cos \theta_{i}=0, \quad i=\overline{1, n},
$$

are nonholonomic constraints. Conditions (2.1) and (2.3) imply that the velocity vector of the center of mass of the link is always aligned with the longitudinal axis of the link.

Let us rewrite the constraint equations in terms of angular velocities and rotation angles

$$
f_{i}=v_{1} \sin \theta_{i}-v_{2} \cos \theta_{i}+a \omega \cos \left(\theta_{i}-\theta\right)+\sum_{k=1}^{i-1} a_{k} \omega_{k} \cos \left(\theta_{i}-\theta_{k}\right)+a_{i, 1} \omega_{i}=0 .
$$

To determine the constants $\lambda_{1}, \ldots, \lambda_{n}$, it is necessary to jointly solve the system of equations $(2.2)$ and the time derivatives of the constraints (2.4). Equations (2.2) define the phase flow on a $2 n$-dimensional space $\mathcal{M}^{2 n}=\left\{\theta_{1}, \ldots, \theta_{n}, \omega_{1}, \ldots, \omega_{n}\right\}$, with the constraints (2.4) being its integrals. The equations of motion of the system under consideration lie on the zero level set of the integrals (2.4).

If the law of motion of the leading car is prescribed, one can simplify the problem by dropping the equations of motion. It is possible to determine the desired components of the vector $\left(\theta_{1}, \ldots, \theta_{n}, \omega_{1}, \ldots, \omega_{n}\right)$ by alternately solving the constraint equations (2.4) without using the equations of motion.

\section{Motion with constant angular velocity}

Let the velocity of the components of the center of mass of the leading car change according to the law

$$
v_{1}=u(t) \cos (\Omega t), \quad v_{2}=u(t) \sin (\Omega t) .
$$

Then the angles of rotation and the angular velocity of the leading car have the form

$$
\theta=\Omega t, \quad \omega=\Omega,
$$


and Eqs. (2.4) take the form

$$
f_{i}=u(t) \sin \left(\theta_{i}-\Omega t\right)+a \omega \cos \left(\theta_{i}-\theta\right)+\sum_{k=1}^{i-1} a_{k} \omega_{k} \cos \left(\theta_{i}-\theta_{k}\right)+a_{i, 1} \omega_{i}=0 .
$$

Thus, we consider here motion with a constant angular velocity.

For convenience, we can go to the angles between the axes of the links $\alpha_{1}=\theta_{1}-\theta$, $\alpha_{2}=\theta_{2}-\theta_{1} \ldots, \alpha_{i}=\theta_{i}-\theta_{i-1}, \ldots$ Then the system (3.1) takes the form

$$
f_{i}=u(t) \sin \left(\sum_{m=1}^{i} \alpha_{m}\right)+a \Omega \cos \left(\sum_{m=1}^{i} \alpha_{m}\right)+\sum_{k=1}^{i-1} a_{k} \omega_{k} \cos \left(\sum_{m=k+1}^{i} \alpha_{m}\right)+a_{i, 1} \omega_{i}=0 .
$$

For convenience, we further assume that $a_{i, 1}=a_{i, 2}=1$. Equations (3.2) can be solved with respect to angular velocities:

$$
\omega_{i}=(-1)^{i}\left(u(t) \sin \left(\sum_{m=1}^{i}(-1)^{m+1} \alpha_{m}\right)+\Omega \cos \left(\sum_{m=1}^{i}(-1)^{m+1} \alpha_{m}\right)\right) .
$$

Using Eqs. (3.3) and considering that the condition

$$
\dot{\alpha}_{i}=\omega_{i}-\omega_{i-1}
$$

is satisfied, we can obtain a closed system with respect to variables $\alpha_{1}, \alpha_{2}, \ldots, \alpha_{n}$ :

$$
\begin{aligned}
& \dot{\alpha}_{1}=-\Omega-u(t) \sin \alpha_{1}-\Omega \cos \alpha_{1}, \\
& \dot{\alpha}_{i}=(-1)^{i} 2 \cos \left(\frac{\alpha_{i}}{2}\right)\left(u(t) \sin \beta_{i}+\Omega \cos \beta_{i}\right), \quad i>1,
\end{aligned}
$$

where $\beta_{i}=\sum_{m=1}^{i-1}(-1)^{m+1} \alpha_{m}+(-1)^{i+1} \frac{\alpha_{i}}{2}$.

Since in general the solution of the resulting system of equations is quite time-consuming, let us consider special cases.

\subsection{Uniform movement in a circle}

Let the leading car move in a circle at a constant velocity $u=$ const. Then, after simplification, in the case of three links including the leading car $(n=2)$, Eqs. (3.4) become

$$
\begin{aligned}
& \dot{\alpha}_{1}=-\Omega-u \sin \alpha_{1}-\Omega \cos \alpha_{1}, \\
& \dot{\alpha}_{2}=u\left(\sin \left(\alpha_{1}-\alpha_{2}\right)+\sin \alpha_{1}\right)+\Omega\left(\cos \left(\alpha_{1}-\alpha_{2}\right)+\cos \alpha_{1}\right)
\end{aligned}
$$

with the specified initial conditions $\alpha_{1}(0)=c_{1}, \alpha_{2}(0)=c_{2}$. This system is autonomous and integrable. The analytical solution has the form

$$
\begin{aligned}
& \alpha_{1}=2 \arctan \left(e^{-u t} \frac{B}{u}-\frac{\Omega}{u}\right), \\
& \alpha_{2}=2 \arctan \left(\frac{A e^{-u t}\left((B-\Omega)^{2}+u^{2}\right)+\left(u^{2}+\Omega^{2}\right)\left(-\Omega+\Omega e^{-u t}+2 B u t e^{-u t}\right)+B^{2} \Omega\left(e^{-2 u t}-e^{-u t}\right)}{u\left(u^{2}+\left(\Omega-B e^{-u t}\right)^{2}\right)}\right),
\end{aligned}
$$

where $B=u \tan \left(c_{1} / 2\right)+\Omega, A=u \tan \left(c_{2} / 2\right)$.

Figures 2 and 3 show graphs of the desired functions for different parameter values. 


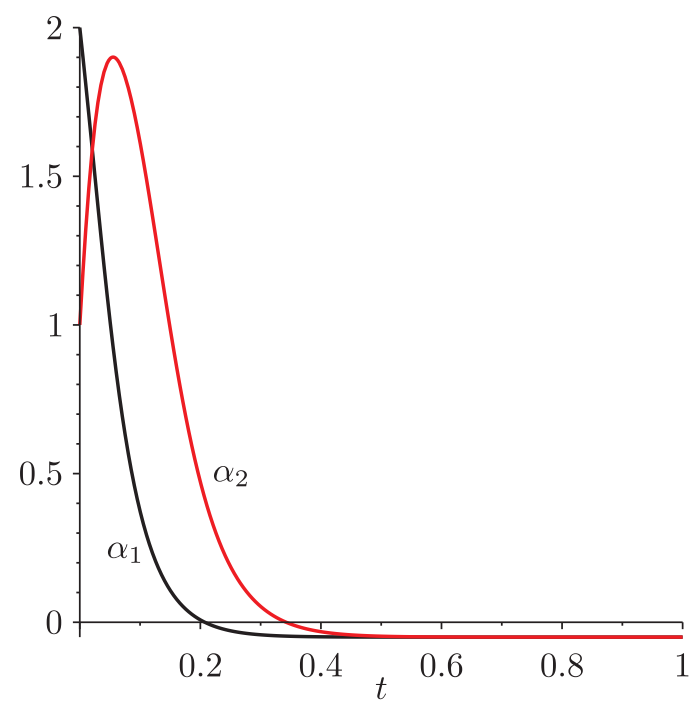

Fig. 2. Graphs of functions $\alpha_{1}(t), \alpha_{2}(t)$ for $u=20$, $\Omega=0.5, c_{1}=2, c_{2}=1$.

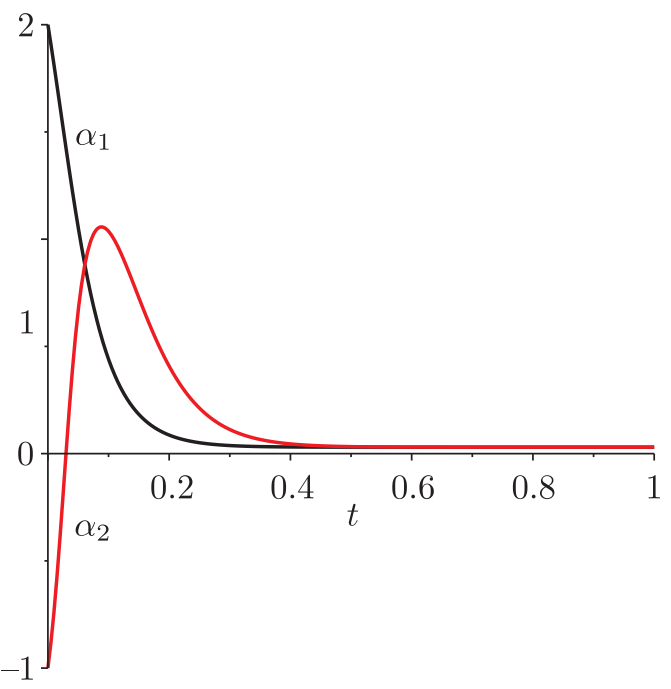

Fig. 3. Graphs of functions $\alpha_{1}(t), \alpha_{2}(t)$ for $u=20$, $\Omega=-0.3, c_{1}=2, c_{2}=-1$.

The desired functions are smooth and the condition $\alpha_{1}(t) \rightarrow 2 \arctan (-\Omega / u)$, $\alpha_{2}(t) \rightarrow 2 \arctan (-\Omega / u)$ for $t \rightarrow \infty$ is true. Therefore, the larger the radius $R=u / \Omega$ of the circle along which the leading car moves, the closer to zero the values of the angles $\alpha_{1}, \alpha_{2}$.

A similar solution will be found with a larger number of links.

\subsection{Curvilinear motion with constant angular velocity}

Let us consider another case when the leading car moves along a certain curve also with a constant angular velocity.

We define the components of the variable velocity

$$
v_{1}=A(2+\sin (\Omega t)) \cos (\Omega t), \quad v_{2}=A(2+\sin (\Omega t)) \sin (\Omega t) .
$$

The trajectory of the leading car for the initial conditions $x_{0}(0)=0, y_{0}(0)=-\frac{2 A}{\Omega}$ is given by the following equations:

$$
x_{0}=\frac{A \sin (\Omega t)(4+\sin (\Omega t))}{2 \Omega}, \quad y_{0}=\frac{A(\Omega t-4 \cos (\Omega t)-\sin (\Omega t) \cos (\Omega t))}{2 \Omega} .
$$

The trajectory is shown in Fig. 4.

The dependence of the velocity on time is shown in Fig. 5.

In the case of three links including the leading car $(n=2)$, the system of differential equations with respect to angles $\alpha_{1}, \alpha_{2}$ takes the form (3.5) for $u=A(2+\sin (\Omega t))$.

In the case of four links including the leading car $(n=3)$, the system of differential equations with respect to angles $\alpha_{1}, \alpha_{2}, \alpha_{3}$ takes the form

$$
\begin{aligned}
& \dot{\alpha}_{1}=-\Omega-u \sin \alpha_{1}-\Omega \cos \alpha_{1}, \\
& \dot{\alpha}_{2}=u\left(\sin \left(\alpha_{1}-\alpha_{2}\right)+\sin \alpha_{1}\right)+\Omega\left(\cos \left(\alpha_{1}-\alpha_{2}\right)+\cos \alpha_{1}\right), \\
& \dot{\alpha}_{3}=-u\left(\sin \left(\alpha_{1}-\alpha_{2}+\alpha_{3}\right)+\sin \left(\alpha_{1}-\alpha_{2}\right)\right)-\Omega\left(\cos \left(\alpha_{1}-\alpha_{2}+\alpha_{3}\right)+\cos \left(\alpha_{1}-\alpha_{2}\right)\right),
\end{aligned}
$$

where $u=A(2+\sin (\Omega t))$.

This system is nonautonomous. The periodic solutions may exist in this system. 


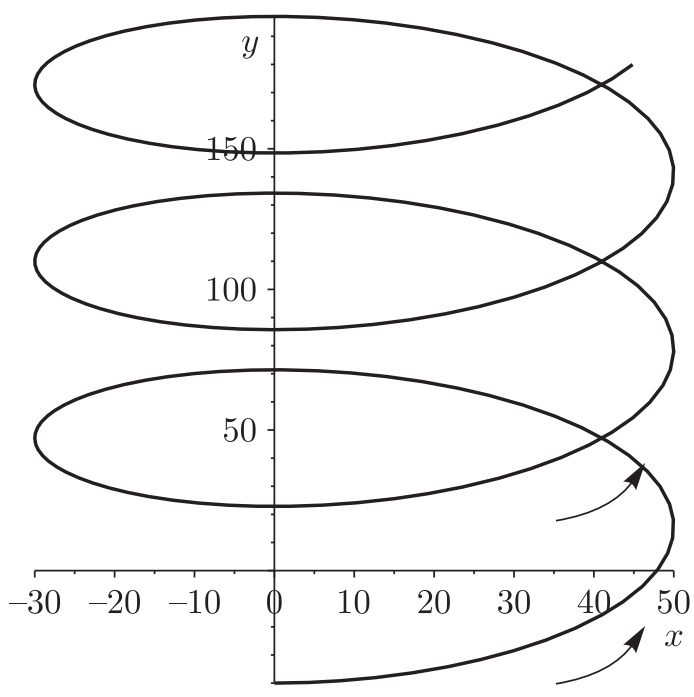

Fig. 4. The trajectory of the leading link for $A=10$, $\Omega=0.5$.

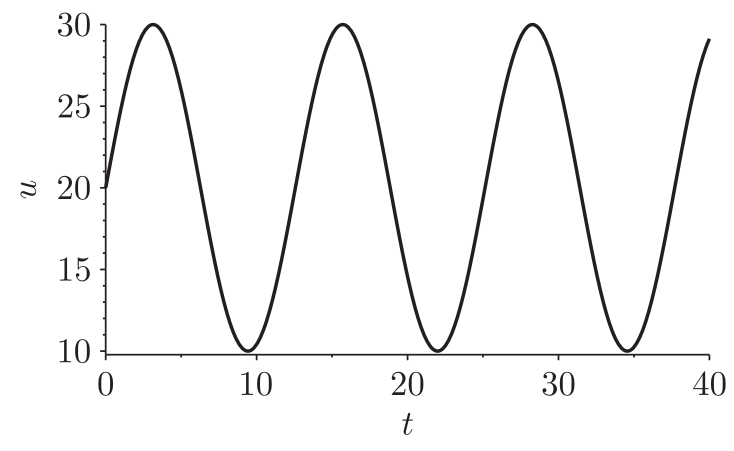

Fig. 5. Speed graph $u$ for $A=10, \Omega=0.5$.

Let us construct a solution of the system for the given numerical parameters. For example, for $A=10, \Omega=-0.5$ and the initial conditions $\alpha_{1}(0)=2, \alpha_{2}(0)=1, \alpha_{3}(0)=-1$, graphs of the unknown functions are shown in Fig. 6.

Clear differences in the behavior of the angle functions are observed at the beginning of the movement. Over time, these functions become periodic. Moreover, over time, the dynamics of the angles does not depend on the initial conditions. But as the link sequence number increases, changes in the behavior of the angle function become more noticeable. For example, for a vehicle consisting of 6 links (including the leading car), the graph of the function $\alpha_{5}$ is shown in Fig. 7 .

For a more detailed analysis of this system (for $u=A(2+\sin (\Omega t))$ ), three-dimensional maps for the period $\frac{2 \pi}{\Omega}$ in space $\alpha_{1}, \alpha_{2}, \alpha_{3}$ were constructed. Numerical experiments showed that, for different values of constants $A$ and $\Omega$, the mappings contain only fixed points. Thus, for different initial conditions, the trajectories are attracted to a certain limit cycle.

Numerical experiments have shown that, with the controlled leading car moving uniformly in a circle, subsequent links occupy a certain limit position over time.

\section{Conclusion}

Two separate problems can be singled out for modeling the dynamics of articulated $n$-trailer wheeled vehicles: the movement of the vehicle when the leading car is under control, and the uncontrolled movement of the vehicle. To describe the dynamics of motion of an uncontrolled wheeled vehicle, it is necessary to jointly solve the equations of motion written in Lagrangian form and nonholonomic constraint equations. And to describe the dynamics of a controlled wheeled vehicle, that is, when the law of motion of the leading car is prescribed, nonholonomic constraint equations are sufficient.

In this work, equations describing the dynamics of a controlled symmetric wheeled vehicle are formulated. Numerical experiments for three-link and four-link wheeled vehicles are constructed. Analytical solutions are obtained for a symmetric design $\left(a_{i, 1}=a_{i, 2}\right)$ for the case of uniform movement of the leading car in a circle. In the case of curved uneven movement, when the linear 

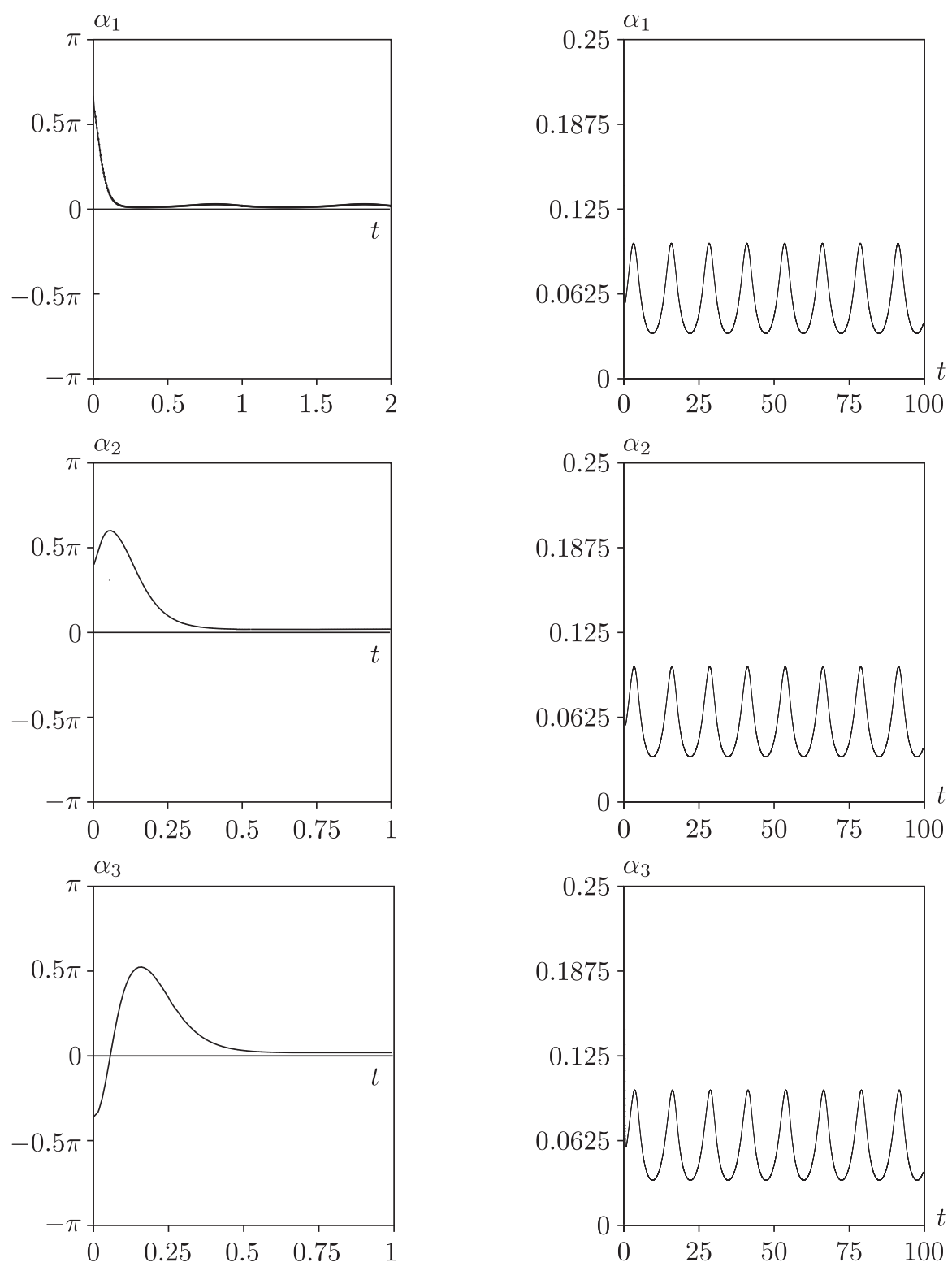

Fig. 6. Graphs of the functions $\alpha_{1}(t), \alpha_{2}(t), \alpha_{3}(t)$.
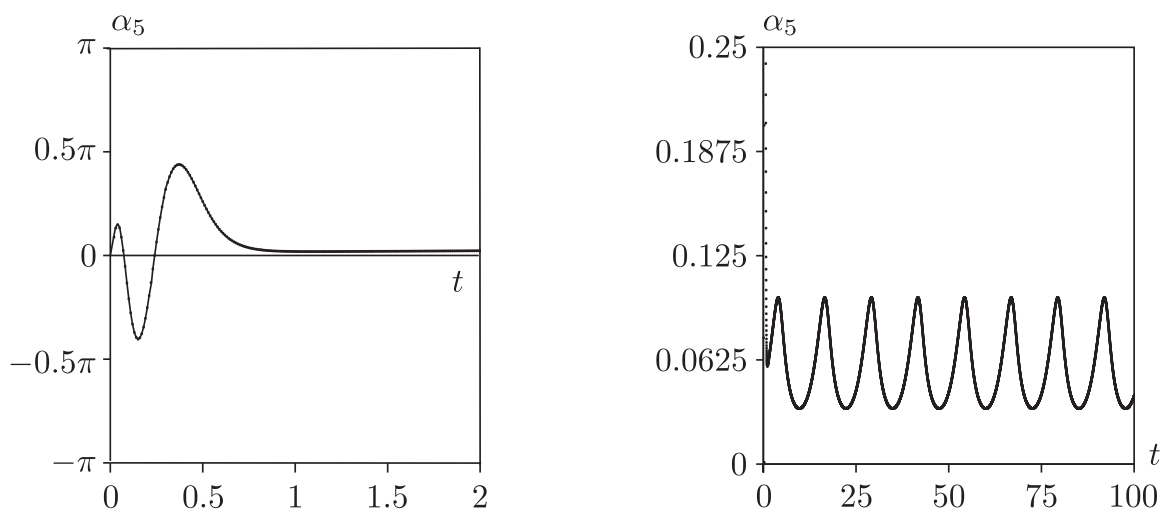

Fig. 7. Graphs of the functions $\alpha_{5}(t)$. 
velocity of the leading car changes periodically, the trajectories are attracted to the limit cycles. In the case under consideration, increasing the number of links does not significantly affect the dynamics of the vehicle. The question of the possibility of the existence of more complex dynamics for an arbitrarily given velocity function remains open. In the cases considered, no chaotic oscillations have been observed.

\section{Acknowledgments}

The author expresses her gratitude to A. V. Borisov for guidance and discussion of the results when working on the article.

\section{References}

[1] Rocard, Y., L'instabilité en mécanique: Automobiles, avions, ponts suspendus, Paris: Masson, 1954.

[2] Lobas, L. G., Nonholonomic Models of Vehicles, Kiev: Naukova Dumka, 1986 (Russian).

[3] Staicu, S., Dynamics Equations of a Mobile Robot Provided with Caster Wheel, Nonlinear Dynam., 2009, vol. 58, no. 1, pp. 237-248.

[4] Martynenko, Yu. G., Motion Control of Mobile Wheeled Robots, J. Math. Sci. (N. Y.), 2007, vol. 147, no. 2, pp.6569-6606; see also: Fundam. Prikl. Mat., 2005, vol.11, no. 8, pp. 29-80.

[5] Laumond, J. P., Controllability of Multibody Mobile Robot, IEEE Trans. Robot. Automat., 1993, vol. 9, pp. 755-763.

[6] Tilbury, D., Murray, R.M., and Sastry, S.Sh., Trajectory Generation for the $n$-Trailer Problem Using Goursat Normal Form, IEEE Trans. Automat. Control, 1995, vol. 40, no. 5, pp. 802-819.

[7] Bravo-Doddoli, A. and García-Naranjo, L. C., The Dynamics of an Articulated $n$-Trailer Vehicle, Regul. Chaotic Dyn., 2005, vol. 20, no. 5, pp. 497-517.

[8] Borisov, A. V., Lutsenko, S. G., and Mamaev, I.S., Dynamics of a Wheeled Carriage on a Plane, Vestn. Udmurtsk. Univ. Mat. Mekh. Komp. Nauki, 2010, no. 4, pp. 39-48 (Russian).

[9] Borisov, A. V., Kilin, A. A., and Mamaev, I. S., On the Hadamard-Hamel Problem and the Dynamics of Wheeled Vehicles, Regul. Chaotic Dyn., 2015, vol. 20, no. 6, pp. 752-766.

[10] Borisov, A. V., Kilin, A.A., and Mamaev, I. S., Invariant Submanifolds of Genus 5 and a Cantor Staircase in the Nonholonomic Model of a Snakeboard, Internat. J. Bifur. Chaos Appl. Sci. Engrg., 2019, vol. 29, no. 3, 1930008, 19 pp.

[11] Grabowski, J., de León, M., Marrero, J.C., and Martín de Diego, D., Nonholonomic Constraints: A New Viewpoint, J. Math. Phys., 2009, vol. 50, no. 1, 013520, 17 pp.

[12] Borisov, A. V. and Mamaev, I. S., The Dynamics of a Chaplygin Sleigh, J. Appl. Math. Mech., 2009, vol. 73, no. 2, pp. 156-161; see also: Prikl. Mat. Mekh., 2009, vol. 73, no. 2, pp. 219-225.

[13] Altafini, C., Some Properties of the General n-Trailer, Internat. J. Control, 2001, vol. 74, no. 4, pp. 409-424.

[14] de Wit, C. C., NDoudi-Likoho, A.D., and Micaelli, A., Nonlinear Control for a Train-Like Vehicle, Int. J. Robot. Res., 1997, vol. 16, no. 3, pp. 300-319.

[15] Campion, G., Bastin, G., and d'Andréa-Novel, B., Structural Properties and Classification of Kinematic and Dynamic Models of Wheeled Mobile Robots, IEEE Trans. Robot. Autom., 1996, vol. 12, no. 1, pp. 47-62.

[16] Pavlovsky, V.E. and Petrovskaya, N. V., Investigation of the Dynamics of Movement of the Chain "Robopoezd": Methods of Planning of Movement, Preprint No. 121, Moscow: KIAM, 2005 (Russian).

[17] Borisov, A. V., Mikishanina, E. A., and Sokolov, S. V. Dynamics of Multi-Link Uncontrolled Wheeled Vehicle, Russ. J. Math. Phys., 2020, vol. 27, no. 4, pp. 433-445.

[18] Borisov, A. V., Kilin, A. A., Mamaev, I. S., and Bizyaev, I. A., Selected Problems of Nonholonomic Mechanics, Izhevsk: R\&C Dynamics, Institute of Computer Science, 2016 (Russian). 